\title{
Analysis and Elaboration of an Air-Coupled Ultrasound Wideband Sensor Array
}

\author{
K. HERMAN AND L. STACH \\ Institute of Telecommunications, Teleinformatics and Acoustics, Wrocław University of Technology \\ Wybrzeże Wyspiańskiego 27, 50-370 Wrocław, Poland
}

(Received October 7, 2010)

\begin{abstract}
In this paper a design process of 64 sensor matrix is described. This study presents adaptation of well known sonar engineering methods of analysis in order to design air operating ultrasound beamforming system. The elaborated system is based on wideband micro electro-mechanical systems. A full digital beamformer with advanced digital signal processing block description is also included. The main goal of the system is direct of arrival of ultrasound wave estimation.
\end{abstract}

DOI: $10.12693 /$ APhysPolA.123.31

PACS: 43.60.Fg, 43.60.Vx, 43.60.-c, 43.60.+d

\section{Introduction}

Air-coupled ultrasound devices for obstacle detection inspired by bats echolocation have been developed since many years. In early 90's a group of scientists from University of Maryland worked on BatMobil design 2 channeled transmitter-receiver system for obstacle detection used by a robot. The intersensor intensity difference (IID) and intersensor time difference (ITD) was used to determine the direction of received echo. A sophisticated VLSI model was applied for data processing and described in the paper [1]. Elaboration of new polymer materials like EMFi piezoelectric polymer foil, was another step in so called biosonar system development process. A broadband sonar system was developed by Peremans (Belgium). He investigated also an artificial pinna shapes and its influence on received echo signal [2]. Broadband frequency modulated echolocation sweeps improve both: range and angular resolution and contains much more information in its spectral components than the constant frequency signal.

The rapid progress in digital signal processing (DSP), especially high speed multichannel analog to digital signal processing, gives us opportunity to use multichannel digital beamforming systems. An example of air operating ultrasonic system for obstacle detection is described in the article [3]. It presents a matrix of 64 electret transducers operating at $18.5 \mathrm{kHz}$ situated on a rectangular grid. Another high resolution solution based on beamforming principle and electrostatic transducers, was developed and described by Webb in the paper [4]. It uses high frequency signals up to $100 \mathrm{kHz}$ which improves range resolution up to $2 \mathrm{~mm}$ and the total error of angular position estimation, which is below 2 degrees. Algorithms development for 3D target reconstruction using air-coupled ultrasound devices and digital beamforming are described in the articles [5-7]. The elaborated and described sensor array and digital beamformer operates in a frequency range of $20-40 \mathrm{kHz}$. Design tips and DSP methods provide us a possibility to estimate the source position with 2 degree angular resolution.

\section{Problem analysis}

The main goal of presented work is to design and elaborate an air operating ultrasound wideband sensor array and complete analog and digital signal processing in order to use digital phase beamforming for source position estimation. The main concept was based on the assumption of a specific array sensor grid. Microphones are situated in a rectangular grid creating two sensor lines with constant intersensor spacing. The horizontal array dimension (number of sensors) is much more higher than the vertical one. Such microphones topology implies high horizontal angular resolutions.

\subsection{Linear array parameters}

The directivity pattern of a $M$-elements linear array is given by Eq. (1) where $D$ is interelement spacing and $\lambda$ is the wavelength. The main lobe width is estimated by parabolic width Eq. (2), see [8]. These two equations describe the most important property of main lobe: shape and width

$$
J(\alpha, M)=\frac{\sin \left(M \pi \frac{D}{\lambda}\left(\sin (\alpha)-\sin \left(\alpha_{k}\right)\right)\right)}{M \pi \frac{D}{\lambda}\left[\sin (\alpha)-\sin \left(\alpha_{k}\right)\right]} .
$$

Main lobe width strongly depends on a steering angle $\phi$, Eq. (2). The beam equation is widely described in the book [9]

$$
\mathrm{PW}=\frac{4 \sqrt{3}}{k d \cos \phi \sqrt{M^{2}-1}} .
$$

One of the key element during the digital phase beamformer designing process is spacing between sensors. In order to make spatial sampling ultrasound frequencies for digital phase beamforming, intersensor spacing needs to be in accordance with equivalent Nyquist criteria for the spatial sampling Eq. (3) described in [8]:

$$
d \leq \frac{\lambda}{2} \text {. }
$$

Wavelength $\lambda$ at $40 \mathrm{kHz}$ is equal to $8.6 \mathrm{~mm}$, which determines maximum distance between sensors under $4.3 \mathrm{~mm}$ in accordance with Eq. (3). The maximum operating frequency determines sensor dimensions and hence matrix 
geometry. Continuing analysis of array property, the array gain Eq. (4) have to be described.

$$
G=10 \log _{10}\left(\frac{\left|\sum_{m=0}^{M-1} w_{m}\right|^{2}}{\sum_{m=0}^{M-1}\left|w_{m}\right|^{2}}\right) .
$$

Array gain with constant sensors weights $w=1$ equals to the number of sensors $M$, Eq. (4).

\subsection{Analysis of analog and digital signal processing}

Starting from PW Eq. (2) and Nyquist criteria Eq. (3), it is necessary to analyze possibilities of spatial sampling a $40 \mathrm{kHz}$ ultrasound wave.

With regard to the limited sampling frequency and the integer number of samples, which correspond to the beam steering angle, the frequency of sampling providing the minimal angular resolution must be multiplied in accordance with Eq. (5) by coefficient $180 /\left(\pi \Phi_{\min }\right)$ :

$$
f_{\mathrm{S}}=f_{\mathrm{SN}} \frac{180}{\pi \Phi_{\min }} \text {. }
$$

Detailed description of a sampling correction could be found in [9]. After conversion (3) with (5) we receive (6):

$$
f_{\mathrm{S}}=\frac{c}{d} \frac{180}{\pi \Phi_{\min }}
$$

Assuming that angular precision of 5 degree using $40 \mathrm{kHz}$ wave, the minimal sampling frequency have to equal about $0.9 \mathrm{MHz}$.

\subsection{Array geometry}

As it was denoted in Sect. 2.1 matrix geometry is determined by sensor size and sensor placement. To determine different ultrasound sources matrix elements have to be broadband. It is recommended to use minimal size sensors with omnidirectional directivity characteristic. In the elaborated solution sensors Knowles Acoustics SPM0204UD5 (Fig. 1) are used.Some of their most important parameters are listed below:

size: $L=4.72 \mathrm{~mm}, W=3.76 \mathrm{~mm}$,

range: $10-65 \mathrm{kHz}$,

sensitivity: $>7 \mathrm{mV} / \mathrm{Pa}$.

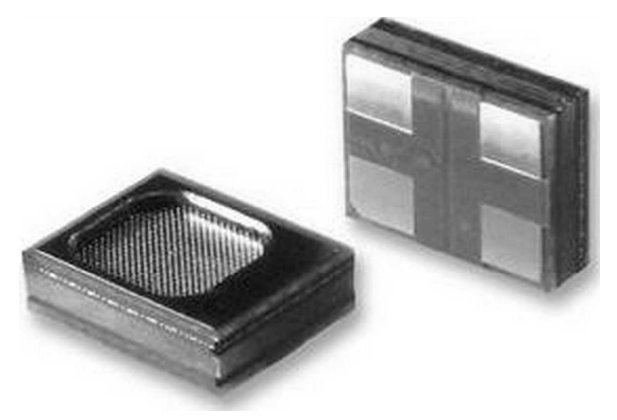

Fig. 1. Knowles Acoustics SPM0204UD5 ultrasonic pressure sensors.

Sensor spatial distribution is planar and states two microphone linear arrays of 32 microphones. The outer dimension of printed circuit board including microphone

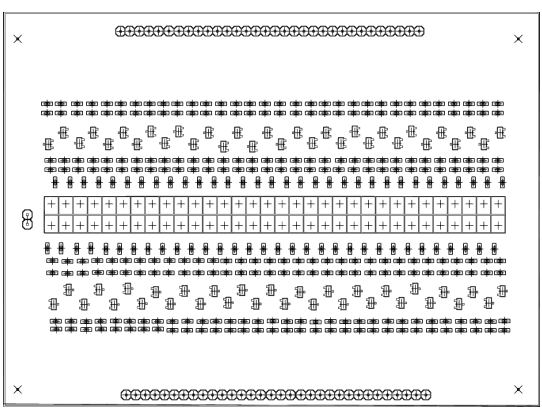

Fig. 2. Printed circuit board top layer layout.

preamplifiers is $140 \times 120 \mathrm{~mm}$ and the sensor spacing is 3.80 and $5.54 \mathrm{~mm}$ in horizontal and vertical plane, respectively. The vertical projection and the arrangement of the elements is shown in Fig. 2. Assuming, the frequency of the sampling $1 \mathrm{MHz}$, it is possible to sample signals in the plane up to the $45 \mathrm{kHz}$ horizontally and $30 \mathrm{kHz}$ vertically.

\subsection{Analog and digital signal routing}

On the block scheme shown in Fig. 3 hardware implementation of proposed solution is presented.Micro electro-mechanical systems (MEMS) array is 64 channel receiver with 64 independent signals. Microphone signals are gained by a block of low-noise preamplifiers. Acquisition of 64 channels by 8 analog to digital converting inputs is realized using time divisionmultiplexing

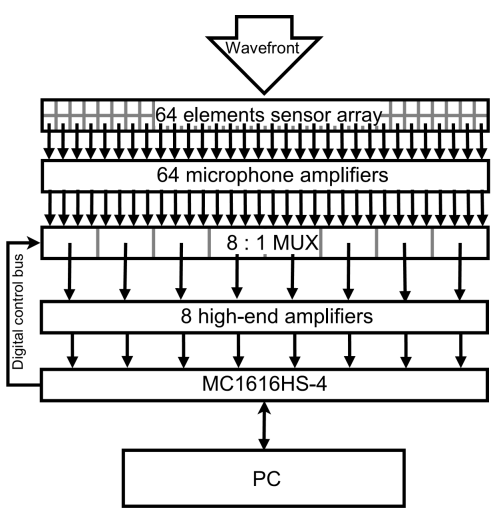

Fig. 3. Full block scheme including analog signal paths, acquisition diagram and digital control signals flow.

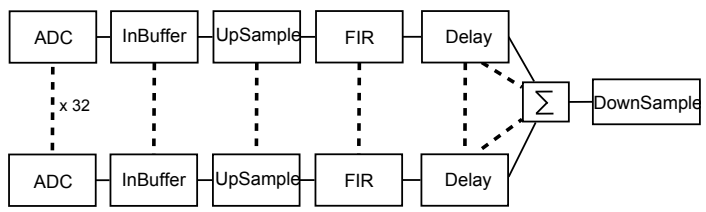

Fig. 4. Digital beamforming of 32 channels with upsampler, FIR interpolation filter digital delay lines, digital summing and downsampling blocks. 
by eight 8:1 multiplexers. Eight currently chosen channels are post gained and recorded by a high speed measurement card MC1616HS-4 DAC. Channel switching is being made in the digital form by the address system which needs only 6 bits. The first 3 ones generate the multiplexer address while another 3 specify the input channel. Signal acquisition is controlled by the software, written in $\mathrm{C} / \mathrm{C}++$ using wxWidgets. Eight analog inputs are sampled simultaneously with sampling frequency of $f_{\mathrm{s}}=1 \mathrm{MHz}$ and 12 bit resolution. After eight measurement cycles 64 channels are exported to PCM wave files. Signal processing is being made offline in MATLAB computational environment. At the first stage the signals are 4 times upsampled and filtered by 20th order FIR interpolation filter. Afterwards the delay and sum beamforming algorithm is being processed as shown in Fig. 4. The sum of beamformer is downsampled 4 times to reduce number of samples.

\section{Matrix measurements}

The directional properties of the matrix were tested during measurement session. Two piezoceramic transducers operating at frequencies $40 \mathrm{kHz}$ and $27 \mathrm{kHz}$ connected to the HP33120A signal generator were tested as sound sources. A burst test signal was generated at the $40 \mathrm{~dB}$ SPL with 20 cycles of signal burst and $50 \mathrm{~Hz}$ burst rate. The measurement setup is shown in Fig. 5 . The horizontal angular sound source position was being changed in an angle range $\pm 32^{\circ}$. The error between estimated angle and real sound source position is shown in Fig. 6.

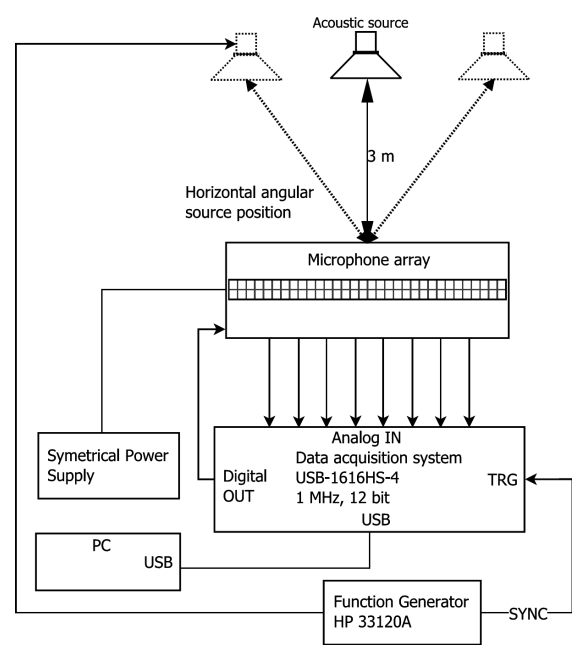

Fig. 5. Horizontal angular resolution measurement setup.

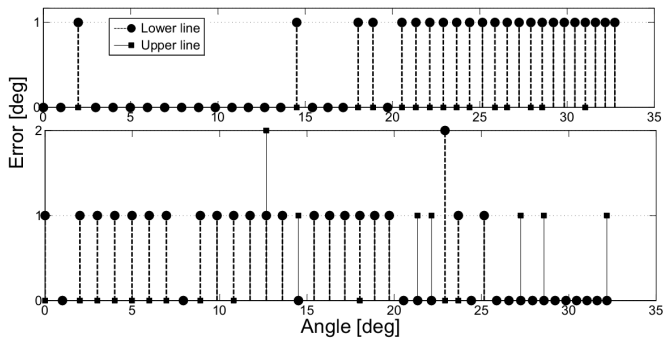

Fig. 6. Differences of horizontal angle estimation using digital beamforming for upper and lower microphone array at $27 \mathrm{kHz}$ and $40 \mathrm{kHz}$ frequencies.

\section{Conclusions}

A full digital air operating ultrasound beamformer was described in the article. The broadband input sensors enable to localize different ultrasound sources with high angular resolution. This property of the sensors supports ability to modify signal processing blockset and apply pulse compression technique to improve the range and angular resolution. Such kind of solution could be an input device for air operating echolocation system with frequency modulated chirp signals commonly used by most of bat species. Minimization of the intersensor spacing provides ability to operate at higher frequencies and, as a result, achieve better range and angular resolution.

\section{References}

[1] R.Z. Shi, T.K. Horiuchi, IEEE Trans. Circuits Syst. 54, $74(2007)$

[2] F. Schillebeeckx, F. De Mey, H. Peremans, in: Proc. 2nd IEEE RAS \&EMBS Int. Conf. on Biomedical Robotics and Biomechatronics BioRob 2008, IEEE, Scottsdale 2008, p. 366 .

[3] M.R. Strąkowski, B.B. Kosmowski, R. Kowalik, P. Wierzba, IEEE Sensor J. 6, 179 (2006).

[4] P. Webb, C. Wykes, IEEE Robotics Automat. 12, 138 (1996)

[5] T.N. Chou, C. Wykes, in: Proc. Sixth Int. Conf. on Image Processing and Its Applications, Vol. 1, Dublin 1997, p. 189.

[6] L. Medina, C. Wykes, Ultrasonics 39, 19 (2001)

[7] C. Wykes, F. Nagi, P. Webb, in: Proc. Int. Conf. on Acoustic Sensing and Imaging, London 1993, p. 77.

[8] D.E. Dudgeon, D.H. Johnson, Array Signal Processing, Prentice-Hall, Englewood Cliffs 1993.

[9] R. Salamon, Hydrolocation Systems, The Scientific Society of Gdańsk, Gdańsk 2006. 